\title{
La vivienda social en Chile. Y la construcción del espacio urbano en el Santiago del siglo $\mathrm{XX}$
}

\author{
Historial de la reseña \\ Recibido: \\ 13 de julio de 2020 \\ Aceptado: \\ 14 de julio de 2020
}

\author{
Florencia Vergara $^{a}$ \\ a Coordinadora de Investigaciones Territoriales. Centro de Estudios e Incidencia Techo - Fundación Vivienda (florencia. \\ vergara@techo.org)
}

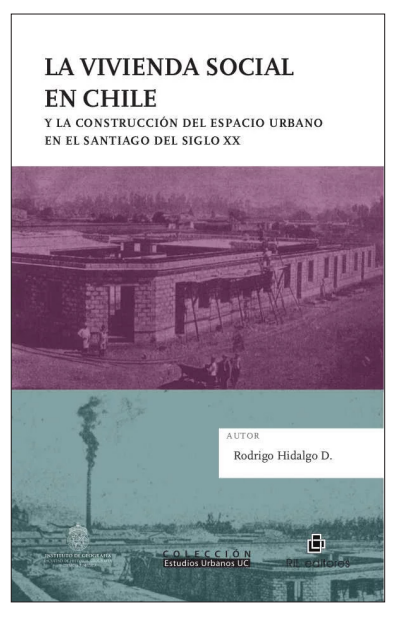

La vivienda social en Chile. Yla construcción del espacio urbano en el Santiago del siglo XX

Rodrigo Hidalgo D.

RIL editores, 2019

538 pág.

ISBN: 978-956-01-0698-0
La pandemia trajo de vuelta al debate público un tema que en la práctica nunca dejó de ser en extremo importante y urgente para gran parte de los chilenos: la cuestión de la vivienda. El momento actual y el llamado a "quedarse en la casa" ha puesto en evidencia que existe una tremenda deuda en términos habitacionales y urbanos, que no sólo involucra los requerimientos por nuevas viviendas sino que se extiende a todos aquellos hogares cuyas viviendas necesitan mejoras, ya sea porque viven hacinados, no tienen acceso a servicios básicos o viven segregados. En este contexto, reseña la reedición del libro de Rodrigo Hidalgo "La vivienda social en Chile. Y la construcción del espacio urbano en el Santiago del siglo XX", originalmente publicado en 2005, y ocurre en un momento crucial, ya que invita a mirar lo contingente de la cuestión de la vivienda desde una perspectiva histórica. A través de una revisión precisa, expone las principales acciones que el Estado desarrolló a lo largo del siglo $\mathrm{XX}$, para dar respuesta al problema de la vivienda, examinando los cambios institucionales y los efectos espaciales producidos a escala urbana.

El autor, Doctor en geografía humana, Director del Doctorado en Geografía de la Pontificia Universidad Católica de Chile y Premio Nacional de Geografía en 2014 ensambla distintas escalas y espacios. Relaciona la vivienda y la ciudad de una manera enriquecedora, mientras nos lleva a imaginar las condiciones de vida en un "cuarto redondo" del año 1890, y al mismo, tiempo nos describe la respuesta del Estado y su efecto en la creación de nuevas periferias. En esencia, el libro demuestra que enfrentar el problema de la vivienda radica en conjugar cantidad y calidad de las soluciones en sus distintas escalas, para avanzar en la construcción no sólo de viviendas adecuadas individualmente, sino también construir de ciudades más justas. Al reconocer las consecuencias de la política habitacional a escala urbana, que hoy se observan en los altos nivel de segregación, concentración de pobreza y acceso deficitario a bienes públicos urbanos, el libro nos empuja a buscar soluciones a través de políticas que contemplen en su diseño los efectos que producen en la ciudad, dejando atrás el sesgo meramente cuantitativo. Esto no significa perder de vista la urgente necesidad de nuevas viviendas, sino más bien es un impulso a innovar desde la política pública para que la vivienda social, sea una forma de mejorar la calidad de vida de las personas.

El objetivo del libro es revisar desde una perspectiva geográfica la política habitacional chilena. En ese sentido, muestra la ciudad actual como resultado de un entramado de acciones y relaciones desarrolladas a través del tiempo, 
una historia con avances pero también con problemas persistentes. Mediante la revisión de las políticas, planes y programas, el libro evidencia una evolución en la forma de entender problema de la vivienda, el rol del Estado (aunque aquí también hay retrocesos) y la institucionalidad dispuesta para enfrentarlo. También deja claro que los problemas persisten, principalmente por la incapacidad de reducir de manera significativa el déficit habitacional, situación preocupante hasta el día de hoy.

A través de diez capítulos se recorre la historia de la vivienda social en Chile durante el siglo XX y de esta manera la transformación de la ciudad de Santiago. El primer capítulo nos introduce a fines del siglo XX, cuando el concepto de vivienda social comienza a utilizarse y se convierte en una preocupación del Estado y las elites por las condiciones precarias en las que habita una parte importante de la población. Desde la aproximación a la vivienda en el marco de la "cuestión social", el libro revisa en un segundo y tercer capítulo las primeras acciones del Estado para enfrentar los problemas habitacionales, destacando la Ley de Habitaciones obreras de 1906 y la Ley de arrendamientos y la normativa de habitaciones baratas de 1925 .

El cuarto capítulo se introduce en las leyes de fomento a la edificación obrera de la década de 1930 y la creación de la Caja de Habitación Popular, donde el Estado asume un rol más protagónico en la construcción de viviendas como respuesta al problema constante del déficit habitacional. El rol más protagónico del Estado toma forma, como muestra el capítulo quinto, con la creación de la Corporación de la Vivienda (CORVI) en el año 1953. En este periodo además se desarrollan los programas de autoconstrucción y los planes habitacionales de los años 50' que materializan grandes conjuntos habitacionales y, con la construcción en altura, transformando la morfología a escala urbana. Transportarse a esta década implica además revisar el surgimiento de la Cámara Chilena de la Construcción, un actor que ha influido de manera significativa en la política de vivienda hasta la actualidad.

Entrando en la década de los 60', el autor trata la Ley de 1959 sobre el Plan Habitacional y el Programa Nacional de Vivienda. En este periodo, destaca el lugar cada vez más importante que ocupan los actores privados, con la incorporación de la vivienda para los sectores de menos recursos en el negocio inmobiliario. Sin embargo, aunque la vivienda social cubre importantes superficies de la capital, aún no se logra hacer frente al déficit. El séptimo capítulo vuelve con fuerza al plano institucional, con la creación del Ministerio de Vivienda y Urbanismo el año 1965. El autor narra un periodo activo donde la demanda por la vivienda adquiere protagonismo, se articulan movimientos sociales y abundan las tomas de terrenos. Esta vez el Estado responde al déficit entregando acceso a la tierra periférica mediante la Operación Sitio, una huella persistente en nuestra ciudad.

El periodo activo de demanda por la vivienda de fines de los 60 ' tiene repercusiones en la década siguiente. El autor aborda en el octavo capítulo las respuestas de principio de los 70' a través de los Planes Anuales de Vivienda y del cambio en la concepción de la vivienda como un derecho del pueblo, que no debe someterse al lucro, y cuya provisión es responsabilidad del Estado. El proceso se interrumpe de forma abrupta con el golpe de Estado, pero como menciona el autor, quedan algunos legados relevantes en la planificación urbana como la importancia de la mixtura social para construir ciudades integradas y justas. 
Ya acercándonos al final, el noveno capítulo marca un punto de quiebre que el autor retrata con detención. La dictadura militar cambia radicalmente la concepción del derecho a la vivienda, supeditándolo al esfuerzo individual y ahorro familiar. Además, se instala una lógica subsidiaria que crea un mercado abierto de la vivienda, donde el Estado sólo interviene mediante subsidios a la demanda y el privado es responsable de la producción. La misma lógica que opera en el ámbito de la vivienda, se reproduce en la planificación urbana. Finalmente, en el décimo capítulo aborda las políticas de vivienda implementadas con la vuelta a la democracia y el desafío que implica nuevamente la reducción del déficit. Durante este periodo la producción de vivienda supera los promedios históricos, sin embargo, este logro cuantitativo es duramente criticado. Como expone el autor, la calidad de las viviendas sociales, sumado a su concentración espacial, generó áreas homogéneas y extensas de pobreza urbana, intensificando los procesos de fragmentación y segregación social.

El principal aporte del libro es que permite mirar en perspectiva los problemas habitacionales y urbanos actuales (algunos de ellos acentuados en medio de la pandemia), y reflexionar con mayor profundidad sobre la necesidad de transformaciones de fondo. La revisión de las políticas de vivienda social en Chile nos muestra, en primer lugar, que muchos de los problemas siguen siendo los mismos. Cuando se exponen las condiciones de habitabilidad precaria de los cuartos redondos de principios del siglo XX, es imposible no pensar en los conventillos ocultos que albergan familias completas en una pieza bajo condiciones abusivas de arriendo y que han salido a la luz en medio de la crisis sanitaria actual. Así, como tampoco, cuando el autor se refiere a asentamientos espontáneos o tomas de terreno en los 70', podemos obviar las cifras que muestran que en la última década las familias que viven en campamentos en Chile han aumentado en un $72 \%{ }^{1}$. Para qué hablar del déficit habitacional, que motiva todas las políticas, pero hasta el día de hoy ha sido una meta imposible de alcanzar. Por otra parte, el libro entrega antecedentes históricos que permiten entender la persistencia de la cuestión de la vivienda y del déficit, dentro de los cuales es relevante destacar: las limitantes institucionales y económicas del Estado a mediados del siglo XX; la inclusión paulatina durante las décadas siguientes de actores privados en la provisión de vivienda; y finalmente, un quiebre en el periodo neoliberal que se traduce en una presencia muy débil del Estado, que da lugar a un mercado cada vez más excluyente y con enormes costos a escala urbana.

Queda hecha la invitación a sumergirse en lo que va del siglo XXI y revisar los cambios en las políticas en el periodo neoliberal. Para comprender las políticas impulsadas en lo que va de este nuevo siglo, será necesario continuar profundizando en el rol cada vez más importante de los actores privados, en el proceso de financiación de la vivienda y en los efectos desiguales en la ciudad. La situación que vivimos actualmente producto de la pandemia y el lugar central que ha ocupado la vivienda en la discusión pública, es un buen punto de partida para continuar este debate. Sin duda, habrá que volver a revisar la historia para extraer aprendizajes, evitar la normalización de este problema, y poder pensar más creativamente en los retos que tenemos por delante. Para que no sea sorpresa la realidad habitacional y urbana precaria en la que vive gran parte de los habitantes de nuestra ciudad, aquí hay más de 100 años de historia.

${ }^{1}$ Bravo, S. (2019). Asentamientos informales en Chile: aumento esperable de un problema conocido. Revista CIS, 16(27), 7-12. Recuperado de https://dialnet.unirioja.es/servlet/ articulo? codigo $=7395758$ 